\section{Risco de exposição à sobrecarga térmica para trabalhadores da cultura de cana-de-açúcar no Estado de São Paulo, Brasil}

\author{
Risk of exposure to heatstroke in sugarcane \\ workers in São Paulo State, Brazil
}

\author{
Riesgo de exposición a la sobrecarga térmica \\ para trabajadores de plantaciones de caña de \\ azúcar en el estado de São Paulo, Brasil
}

\section{Resumo}

O calor ambiental é um agravante às atividades dos trabalhadores da cultura de cana-de-açúcar, sendo o corte manual uma das atividades pesadas que favorece a elevada geração de calor metabólico. Isso associado com outras condições precárias de trabalho favorece o estresse térmico, podendo provocar graves doenças ou até levar à morte. O objetivo deste estudo é quantificar o risco de sobrecarga térmica que os cortadores de cana-de-açúcar de São Paulo, Brasil, são expostos. Por meio de metodologia específica, foram utilizados quatro anos de dados para estimar o Índice de Bulbo Úmido - Termômetro de Globo sob a consideração das atividades leve, moderada e pesada. Os resultados mostram extrapolação do limite legal de exposição para atividade contínua em períodos de 1 a 8 horas, o que requer medidas de controle para continuidade do trabalho. A principal constatação é a observância do risco de sobrecarga térmica para os três tipos de atividade: leve, moderada e pesada. Os limites de tolerância são ultrapassados durante todo o turno de trabalho em cerca de $7 \%$ dos dias, para atividade pesada, e em cerca de 3\%, para atividade moderada. Além disso, percebeu-se que as áreas com maior risco de sobrecarga térmica são as mesmas onde há predominância da cultura de cana-de-açúcar.

Transtornos de Estresse por Calor; Trabalhadores Rurais; Saccharum
Rodrigo Cauduro Roscani 1

Daniel Pires Bitencourt 2

Paulo Alves Maia 1

Alvaro Cesar Ruas 1

doi: 10.1590/0102-311X00211415

\author{
Correspondência \\ D. P. Bitencourt \\ Fundação Jorge Duprat Figueiredo de Segurança e Medicina do \\ Trabalho. \\ Rua Silva Jardim 213, Florianópolis, SC 88020-200, Brasil. \\ daniel.bitencourt@fundacentro.gov.br \\ 1 Fundação Jorge Duprat Figueiredo de Segurança e Medicina \\ do Trabalho, Campinas, Brasil. \\ 2 Fundação Jorge Duprat Figueiredo de Segurança e Medicina \\ do Trabalho, Florianópolis, Brasil.
}




\section{Introdução}

O Brasil é hoje o maior produtor mundial de cana-de-açúcar e de seus derivados, açúcar e etanol. Essa cultura, introduzida no período colonial, representa, atualmente, um dos principais setores da economia agrícola nacional 1. Em 2009, segundo o Instituto Brasileiro de Geografia e Estatística (IBGE), a produção nacional foi de 687 milhões de toneladas de cana-de-açúcar ${ }^{2}$. Naquele ano, o setor sucroalcooleiro empregou cerca de quatro milhões de trabalhadores ${ }^{3}$. Na safra de 2014, o IBGE estimou uma produção de 738 milhões de toneladas 2, dos quais o Estado de São Paulo é responsável por 55,8\%.

A crescente mecanização da colheita em São Paulo, decorrente da Lei no 11.241/02 4, tem foco ambiental e não de preservação da segurança e saúde do trabalhador. Em 2012, a mecanização atingiu, aproximadamente, 73\% da área plantada. Apesar disso, em 2013, cerca de 200 mil trabalhadores atuaram no corte manual da cana-de-açúcar, predominantemente nas pequenas propriedades. Também se deve considerar que há áreas não mecanizáveis devido a inclinações maiores que $12^{\circ}$. Além disso, há a necessidade de a primeira colheita ser manual para não prejudicar a rebrota 5 . Portanto, a total mecanização da colheita em São Paulo parece não se apresentar como uma realidade próxima.

As condições de trabalho oferecidas aos trabalhadores da cultura de cana-de-açúcar não são adequadas, e isso é percebido há muitas décadas. O trabalho infanto-juvenil nos canaviais, já identificado como um grave problema na segunda metade da década de 1990 por Gomes et al. 6, ainda é uma realidade por volta do ano de 2010 7. O emprego formal tem crescido em São Paulo, mas em índices muito aquém ao aumento da produção. A produtividade média diária dos cortadores de cana-de-açúcar passou de 3 toneladas, na década de 1950, para 6 toneladas, na década de 1980, e 12 toneladas, no final da década de 1990 8. Normalmente, esses trabalhadores desenvolvem suas atividades sob péssimas condições de trabalho. A poluição no ar provocada pelo processo de queima da cana-de-açúcar, por exemplo, além de causar impactos ambientais ${ }^{9}$, também favorece a ocorrência de problemas respiratórios nos trabalhadores e na população ao redor do cultivo 10,11. Além disso, de acordo com Bitencourt et al. 12, esses trabalhadores, na maioria das vezes, alimentam-se mal, são transportados de forma inadequada, não têm controle do processo de reidratação durante a jornada de trabalho e descansam apenas poucos minutos após a alimentação. Os trabalhadores da cana-de-açúcar não dispõem de todos os equipamentos de proteção individual e frequentemente improvisam para evitar danos físicos. O local do cultivo geralmente não possui equipamentos de primeiros socorros, condições mínimas de higiene e instalações sanitárias.

A cada jornada de trabalho, os cortadores de cana-de-açúcar realizam 3.792 golpes de facão, exercendo 3.994 flexões de coluna para produzir cerca de 11 toneladas de cana-de-açúcar 13. Um dos aspectos mais graves nesse processo é a remuneração por produção, abordada em vários trabalhos 7,14, 15,16,17,18. A expectativa do ganho financeiro leva o trabalhador a ignorar os alertas fisiológicos, podendo ocasionar a exaustão e consequentes danos agudos e crônicos à sua saúde. Esse risco é acentuado pela competitividade estimulada entre os colegas e pela pressão hierárquica 8 . Alessi \& Navarro 14 destacam a extensa jornada de trabalho, a intensificação do ritmo de trabalho, o decréscimo real do valor dos salários e o descumprimento dos direitos trabalhistas como fatores marcantes nas características gerais do trabalho no campo. A troca térmica entre o corpo dos trabalhadores e o ambiente é prejudicada pela vestimenta típica utilizada no cultivo da cana-de-açúcar, podendo agravar o estado de estresse ao calor. Apesar de as roupas possuírem a finalidade de proteção e serem necessárias a determinados tipos de trabalho, podem restringir a perda de calor por evaporação 19,20.

Para a manutenção do funcionamento saudável do organismo, é necessário que ocorra o equilíbrio do calor gerado e recebido do ambiente com o dissipado, mantendo a temperatura do corpo em valores normais 21. A sobrecarga térmica ocorre quando há desequilíbrio térmico do corpo, isto é, recebe mais calor do que pode dissipar, causando seu aquecimento acima de $38^{\circ} \mathrm{C}$. Quanto maior a temperatura do corpo, maiores são os efeitos fisiológicos provocados pelo calor. A permanência em condições térmicas desfavoráveis pode causar o estresse térmico, resultando em debilidade do estado geral de saúde, alterações das reações psicossensoriais e queda de produção 22 .

Há estudos que analisam os agravos ou alterações nas respostas fisiológicas de órgãos ou sistemas do corpo humano, relacionados à exposição ao calor 17,19,23,24,25,26. Outros trabalhos associam o calor com aumento da taxa de morbidade e mortalidade 27,28 . No caso de atividade laboral, dependendo das 
características individuais de cada trabalhador, as doenças do calor podem se manifestar por meio de sintomas comportamentais, tais como irritabilidade, confusão mental, câimbras, fadiga severa repentina e outros. Quanto maior a quantidade de atividade física, maior a quantidade de calor gerada 29. Quantitativamente, a eficiência energética muscular é da ordem de $20 \%$, ou seja, produz-se $5 \mathrm{~kW}$ de calor a cada $1 \mathrm{~kW}$ de trabalho realizado 30 . No caso de falência do sistema termorregulador do corpo, há risco de morte, conforme abordado por Bitencourt et al. 12 e Paixão \& Nogueira 31. E deve-se ainda considerar que existe claro risco de estresse térmico mesmo quando os trabalhadores não exercem atividades físicas extremas, somente pelas condições de clima 32 .

Há evidências de que a exposição ao calor associada à atividade representa um risco laboral significativo, sendo inclusive responsável por inúmeras mortes 12,31. Apesar disso, há poucos estudos relacionando o efeito do calor sobre os trabalhadores que exercem atividades específicas, sobretudo no caso dos cortadores da cana-de-açúcar. Contudo, para outras atividades, existem alguns trabalhos relacionando a exposição laboral ao calor com os agravos à saúde. Na Tailândia, por exemplo, Tawatsupa et al. 23 observaram uma relação do calor com as doenças renais. Sett \& Sahu 25 relacionaram os efeitos do calor com o decaimento da produtividade no sistema produtivo de tijolos na Índia. No Canadá, Jay \& Kenny 19 caracterizaram os riscos de exaustão com possibilidade de choque térmico pelo aumento da temperatura interna do corpo por volta de $3^{\circ} \mathrm{C}$.

Buscando entender quantitativamente a realidade da exposição ao calor por parte dos trabalhadores do cultivo de cana-de-açúcar, objetiva-se, neste artigo, calcular e analisar, espacialmente para o Estado de São Paulo, o risco de sobrecarga térmica que esses trabalhadores foram expostos durante um período de quatro anos. Para tanto, apresentam-se análises espaciais e pontuais da frequência de dias em que o Índice de Bulbo Úmido - Termômetro de Globo (IBUTG) ultrapassa o limite de tolerância para as atividades leve, moderada e pesada.

\section{Metodologia}

\section{Área de estudo e dados meteorológicos}

O cultivo de cana-de-açúcar é realizado predominantemente nas porções central, norte e oeste do Estado de São Paulo, conforme disponibilizado em http://www.dsr.inpe.br/laf/canasat/cultivo.html. Entretanto, neste estudo, os cálculos e o mapeamento do IBUTG são realizados para todo o Estado de São Paulo. Os dados meteorológicos utilizados na estimativa do IBUTG são temperatura do ar (T), temperatura do ponto de orvalho $\left(T_{d}\right)$, pressão atmosférica $(p)$, radiação solar global $(R)$ e velocidade do vento $(V)$. Esses dados são observados pela rede de estações meteorológicas de superfície do Instituto Nacional de Meteorologia (INMET), distribuídas em 31 localizações, conforme apresentado na Figura 1. As estações meteorológicas são automáticas e fornecem dados atmosféricos a cada hora.

\section{Estimativa do IBUTG e método de análise}

O IBUTG é o principal parâmetro de análise para a quantificação do estresse térmico. Esse índice foi desenvolvido por Yaglou \& Minard 33, inicialmente, para estudar as relações entre o calor e suas consequências fisiológicas durante treinamentos militares. É o índice legal apresentado na Norma Regulamentadora de Segurança e Higiene do Trabalho 15 (NR-15) 34 e também foi adotado pela International Organization for Standardization (ISO) 7243 35, tendo aprovação mínima de $75 \%$ dos países membros da ISO. A utilização do IBUTG, na análise de sobrecarga térmica laboral, pode ser identificada em estudos de diversas regiões do mundo, tais como na Costa Rica 32 ou no Sudeste Asiático 36.

O IBUTG para ambientes externos com carga solar é dado por:

$$
\text { IBUTG }=\left(0,7 \cdot T_{n}\right)+(0,1 \cdot T)+\left(0,2 \cdot T_{g}\right)
$$

em que $T_{n}$ é a temperatura de bulbo úmido "natural", $T_{g}$ é a temperatura de globo, e $T$ é a temperatura do ar.

Os limites de tolerância (Tabela 1) para trabalhadores expostos ao calor são estabelecidos pela NR-15, a qual define que as medições do IBUTG sejam feitas no local onde o trabalhador executa suas atividades 34 . Quando o IBUTG ultrapassa valores de $30^{\circ} \mathrm{C}$ (atividade leve), 26,7 ${ }^{\circ} \mathrm{C}$ (atividade 
Figura 1

Estado de São Paulo, Brasil, com a localização das estações meteorológicas de superfície do Instituto Nacional de Meteorologia (INMET).

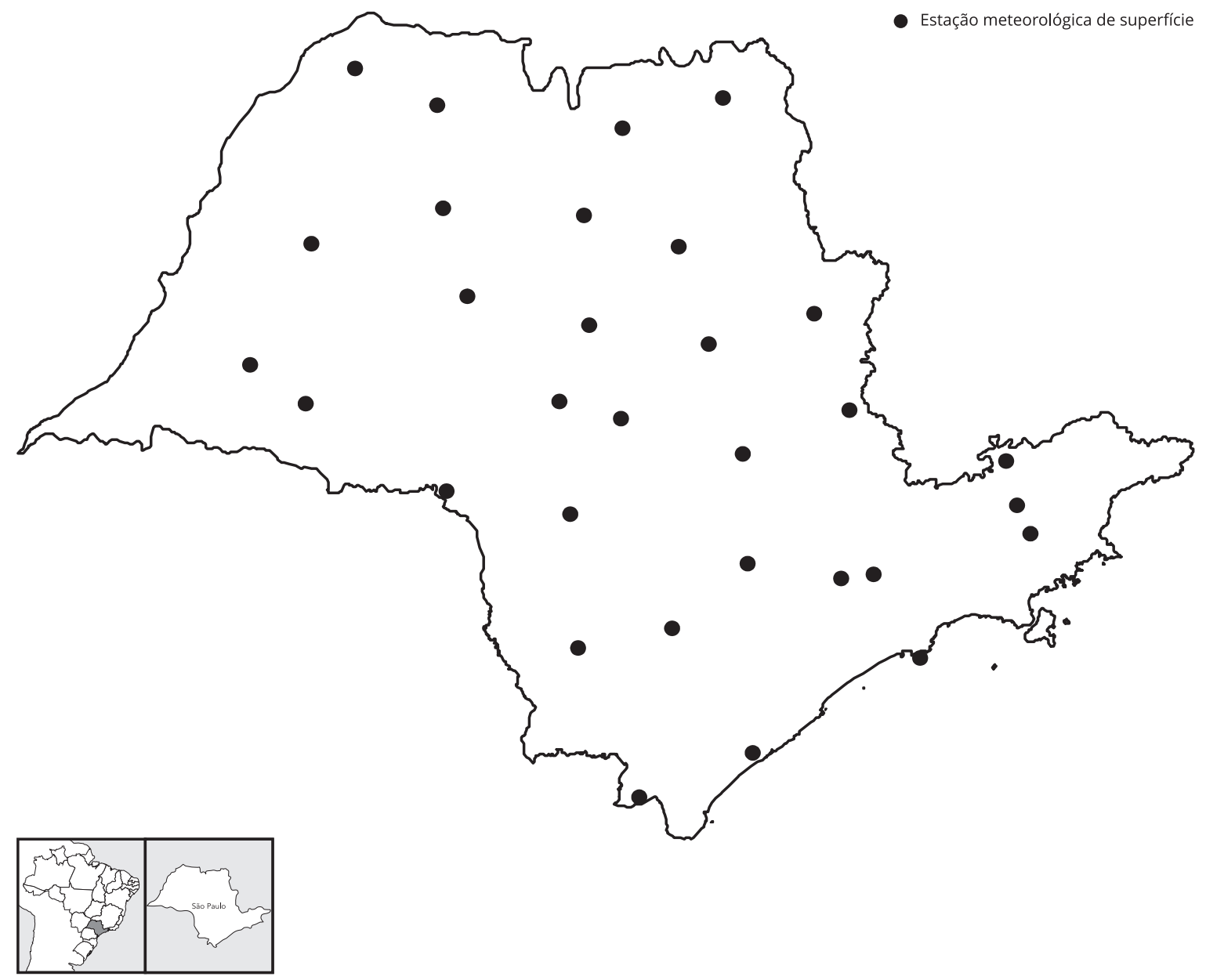

moderada) ou $25^{\circ} \mathrm{C}$ (atividade pesada), tornam-se necessárias medidas preventivas no trabalho para evitar doenças ocupacionais. Acima desses valores (linhas 2, 3 e 4 da Tabela 1), os intervalos de IBUTG correspondem a períodos maiores de descanso, podendo alcançar 15, 30 ou 45 minutos de descanso a cada hora trabalhada. Nas situações mais extremas, quando o IBUTG ultrapassa os limites de $32,2^{\circ} \mathrm{C}$, $31,1^{\circ} \mathrm{C}$ e $30^{\circ} \mathrm{C}$, para atividades leve, moderada e pesada, respectivamente, a condição deve ser entendida como de risco grave e eminente. Para essa condição, não é permitido o trabalho sem adoção de medidas especiais de controle. Ressalta-se ainda que os trabalhadores da cultura de cana-de-açúcar geralmente trabalham em regime contínuo, por períodos maiores que uma hora, sem gozar de descanso térmico necessário para o resfriamento do corpo. Nesse caso, os limites de tolerância a serem considerados para a avaliação da exposição térmica são os da primeira a última linha da Tabela 1 .

Lembra-se que os limites de tolerância são definidos para o pagamento ou não de adicional de insalubridade, enquanto não se adotam medidas de controle. Por outro lado, a origem do IBUTG não foi baseada em estudos sobre a saúde do trabalhador e, portanto, não é fronteira entre a saúde e o adoecimento. As mortes no campo para essa atividade, conforme relatado por Bitencourt et al. 12, talvez sejam indicativos disso. Ainda que não sejam, são notórias as péssimas condições de trabalho 
Quadro 1 do Anexo 3 da Norma Regulamentadora 15 (NR-15) que descreve os limites de tolerância (IBUTG, em $\left.{ }^{\circ} \mathrm{C}\right)$ para exposição ao calor.

\begin{tabular}{|c|c|c|c|}
\hline \multirow[t]{2}{*}{ Regime de trabalho intermitente com descanso no próprio local de trabalho (por hora) } & \multicolumn{3}{|c|}{ Tipo de atividade } \\
\hline & Leve & Moderada & Pesada \\
\hline Trabalho contínuo & até 30,0 & até 26,7 & até 25,0 \\
\hline 45 minutos de trabalho/15 minutos de descanso & 30,1 a 30,6 & 26,8 a 28,0 & 25,1 a 25,9 \\
\hline 30 minutos de trabalho/30 minutos de descanso & 30,7 a 31,4 & 28,1 a 29,4 & 26,0 a 27,9 \\
\hline 15 minutos de trabalho/45 minutos de descanso & 31,5 a 32,2 & 29,5 a 31,1 & 28,0 a 30,0 \\
\hline \multirow[t]{2}{*}{ Não é permitido o trabalho sem adoção de medidas de controle } & acima de & acima de & acima de \\
\hline & 32,2 & 31,1 & 30,0 \\
\hline
\end{tabular}

Fonte: Ministério do Trabalho e Emprego (NR-15 - atividades e operações insalubres) ${ }^{34}$.

as quais esses trabalhadores são submetidos, principalmente se considerarmos o poder econômico do setor sucroalcooleiro.

A Norma Regulamentadora 09 (NR-09) 37, ao abordar o Programa de Prevenção de Riscos Ambientais (PPRA), recomenda monitoramento sistemático e repetitivo. Embora, por vezes, exista alguma complexidade dos cenários e situações sob as quais os trabalhadores executam suas tarefas 38, espera-se que o IBUTG seja monitorado conforme determina a norma. Entretanto, é senso comum que a maioria das empresas realiza apenas monitoramentos esporádicos ou, na melhor das hipóteses, por períodos de, no máximo, três dias. Em geral, a frequência dessas medidas são anuais, sendo executadas por empresas terceirizadas para elaboração de laudos. Daí, surge a importância da utilização de métodos de estimativa dos valores de IBUTG, calculados a partir de outras variáveis ambientais observadas, operacionalmente, por redes de estações meteorológicas. O IBUTG estimado pode ser comparado com os valores observados em campo, além de subsidiar a ação do estado na vigilância das condições de trabalho, fornecer informações prévias sobre as áreas e épocas do ano de maior risco térmico ou disponibilizar um histórico do comportamento do IBUTG para fins de pesquisa ou ações trabalhistas.

Existem muitos métodos de estimativa do IBUTG propostos na literatura e testados em várias partes do mundo 39,40,41,42,43,44,45,46,47. Neste estudo, utiliza-se o método proposto recentemente por Maia et al. 48, o qual utilizou dados meteorológicos observados no Estado de São Paulo para o seu desenvolvimento. A formulação é baseada no método proposto por Hunter \& Minyard 41. Uma das principais adequações apresentadas por Maia et al. 48 foi a consideração de variação da fração de radiação direta do sol $\left(f_{d b}\right)$ e da fração de radiação difusa $\left(f_{d i f}\right)$, parâmetros considerados constantes por Hunter \& Minyard 41. Segundo Maia et al. 48, essa consideração garante uma maior precisão na estimativa do IBUTG, pois contabiliza os efeitos da nebulosidade, situação que comumente ocorre em dias quentes no Estado de São Paulo.

Os valores de $T$ são diretamente observados pelas estações meteorológicas do INMET, enquanto que $T_{n}$ e $T_{g}$ são estimados por meio de expressões matemáticas, em que $T_{n}$ é obtido por:

$$
T_{n}=15,14587+(0,00047711 . R)-(0,56900 . V)+\left(0,45258 . T_{d}\right)
$$

e $T_{g}$ é obtido por:

$0,115 \cdot U^{0,58} \cdot T_{g}+\varepsilon \cdot \sigma \cdot T_{g}{ }^{4}=A \cdot R \cdot\left[f_{d b} \cdot F+\left(1+\alpha_{g r}\right) \cdot f_{d i f}\right](3)+\varepsilon_{a} \cdot\left(1-\alpha_{g}\right) \cdot \sigma \cdot(T+273)^{4}+0,115 \cdot U 0,58 \cdot T$

sendo $U$ a velocidade do vento $(\mathrm{m} / \mathrm{h})$ observada pela estação meteorológica do INMET e corrigida da altura de $10 \mathrm{~m}$ para $1,5 \mathrm{~m}$, ou seja, $U=V(1,5 \mathrm{~m} / 10 \mathrm{~m})^{0,21}, \varepsilon$ é a constante de emissividade térmica do globo, $\sigma$ é a constante de Stefan-Boltzmann, $A$ é o coeficiente de absorção de radiação do globo, $F$ é o fator de forma do globo, $\alpha_{g r}$ é o albedo do solo, $\varepsilon_{a}$ é a emissividade térmica da atmosfera, a qual é uma função da temperatura do ponto de orvalho $\left(T_{d}\right)$, da temperatura do ar $(T)$ e da pressão atmosférica $(p)$, sendo $T_{d}$, $T$ e $p$, juntamente com a variável $R$, observados pelas estações meteorológicas do INMET.

Os procedimentos para obtenção de $f_{d b}$ e $f_{d i f}$, assim como todos os demais detalhes para a estimativa do IBUTG utilizada neste trabalho, podem ser encontrados em Maia et al. 48. Todos os cálculos para estimativa do IBUTG são realizados por meio de um software operacionalizado no Centro 
Técnico Nacional (CTN) da Fundação Jorge Duprat Figueiredo de Segurança e Medicina do Trabalho (FUNDACENTRO). Esse software possui uma interface de acesso público no endereço eletrônico http://www.fundacentro.gov.br/sobrecarga-termica/inicio.

A matriz de IBUTG foi construída com pontos de latitude e longitude igualmente espaçados a cada $40 \mathrm{~km}$. Os valores de IBUTG são atribuídos para cada um desses pontos utilizando os dados atmosféricos observados pelas estações meteorológicas localizadas dentro de um raio de $80 \mathrm{~km}$. Assim, a estimativa pontual do IBUTG pode tomar, como base, observações de mais de uma estação meteorológica, sendo o valor final obtido por meio de interpolação espacial. No caso da $T$, também é feito uma correção para as diferenças de altitude, por meio do lapse rate ambiental. Foram considerados para análise somente os pontos localizados nos limites do Estado de São Paulo, totalizando 137 pontos.

Os cálculos são realizados para todos os dias do período de 1o de setembro de 2010 a 31 de agosto de 2014. E, para cada dia, obtém-se o valor de IBUTG a cada hora, entre 9 e 16 horas (desconsiderando o horário brasileiro de verão). O valor de IBUTG estimado para cada hora cheia é representativo dos 60 minutos anteriores. Portanto, a análise neste estudo é efetivamente realizada para um turno de 8 horas, compreendido entre 8 e 16 horas. Esse período é o turno normalmente utilizado pelos trabalhadores da cultura de cana-de-açúcar para o desenvolvimento de suas atividades.

A análise final é realizada por meio de mapas de frequência. Assim, para cada ponto, é calculado o percentual de dias com impedimento de trabalho contínuo, durante 1, 2, 3, 4, 5, 6, 7 e 8 horas consecutivas, considerando trabalhos do tipo leve, moderado e pesado. $\mathrm{O}$ tipo de atividade é classificado conforme o calor metabólico gerado, o qual pode ser obtido por diversos métodos, tais como consumo de oxigênio, batimento cardíaco, decomposição de movimentos na execução da tarefa ou por meio de tabelas. Neste artigo, considera-se atividade pesada aquela que despende mais que $440 \mathrm{Kcal} / \mathrm{h}$, conforme Quadro no 3 da NR-15 34. A atividade de corte manual de cana-de-açúcar, por meio da decomposição de movimentos, por exemplo, despende $532 \mathrm{Kcal} / \mathrm{h}$ (ISO 8996) 49.

O percentual de dias $\left(P_{d(n)}\right)$ no ponto $n$, com $\mathrm{n}=1,2, \ldots, 137$, é dado pela expressão:

$$
P_{d(n)}=\left[N_{d(n)} / N_{(n)}\right] \times 100
$$

em que $N_{d(n)}$ é o número de dias com impedimento de trabalho contínuo no ponto $n$, e $N_{(n)}$ é o número total de dias que o IBUTG foi estimado no ponto $n$. O $N_{(n)}$ depende da disponibilidade dos dados meteorológicos contidos na série de dados. Os pontos com mais de $15 \%$ de dias faltantes foram desconsiderados. A análise final dos resultados é realizada pelo mapeamento de $P_{d(n)}$ no Estado de São Paulo. Esse mapeamento consiste na plotagem em isolinhas realizada por meio do software Grid Analysis and Display System (GrADS; http://cola.gmu.edu/grads/).

\section{Resultados e discussões}

A vestimenta utilizada pelos trabalhadores da cultura de cana-de-açúcar exerce um importante papel para a condição de estresse térmico. A vestimenta atua como uma barreira à troca de calor entre o corpo e o ambiente, dificultando ou impedindo o resfriamento. A eficiência dessa troca de calor depende de vários fatores, entre eles, o tipo e cor do tecido. Por isso, algumas legislações e normas internacionais recomendam ajustes nos valores de IBUTG para diminuir os limites de tolerância, de acordo com o tipo de vestimenta utilizado na atividade laboral. A Associação Americana de Higiene Industrial, nesses casos, recomenda a diminuição do IBUTG em $2^{\circ} \mathrm{C}$, o que tornaria ainda mais crítica a situação de exposição ao calor. No entanto, a legislação brasileira não leva, em conta, a vestimenta utilizada pelo trabalhador. Desde a sua criação, em 1978, a NR-15 não foi atualizada nesse aspecto. Assim, todas as faixas de valores do IBUTG, utilizadas para os cálculos de frequência e discutidas nessa seção, não foram definidas sob a consideração da vestimenta do trabalhador, seguindo estritamente os valores previstos na NR-15.

Considerando o tipo de atividade leve, o limite de tolerância nunca é ultrapassado durante períodos de 2 a 6 horas consecutivas nas áreas de maior altitude (Figuras 2 e 3; em especial 2d, 2g, 2j, 3a e 3d) e durante períodos de 7 a 8 horas consecutivas em áreas mais abrangentes de São Paulo (Figuras $3 g$ e 3j). Percebe-se ainda que, considerando o tipo de atividade moderada, o limite de tolerância nunca é ultrapassado durante 8 horas consecutivas na região da Serra da Mantiqueira (Figura 3k). No entanto, em todas as outras situações, ou seja, para qualquer tipo de atividade e qualquer período de tempo 
considerado, foi verificado algum nível de risco de sobrecarga térmica no Estado de São Paulo. No caso de atividade leve, a frequência máxima (entre 6 e 9\%) de dias com o IBUTG superando o limite de tolerância durante 1 hora ocorre no norte de São Paulo (Figura 2a).

Considerando atividade moderada, a frequência de dias em que o IBUTG ultrapassa o limite de tolerância em período de 1 hora é superior a 15\%. Esse máximo ocorre no norte do estado, enquanto que, na maior parte das demais áreas, esses valores de frequência ficam entre 9 e 15\% (Figura 2b). Conforme se contabiliza maiores períodos ( 2 a 7 horas) com o IBUTG ultrapassando o limite de tolerância para atividade moderada, a frequência de dias com essa situação passa a ser cada vez menor (Figuras $2 \mathrm{e}, 2 \mathrm{~h}, 2 \mathrm{k}, 3 \mathrm{~b}, 3 \mathrm{e}$ e $3 \mathrm{~h}$ ). Contudo, os maiores valores de frequência sempre ocorrem nas áreas do centro, norte e oeste de São Paulo. Observa-se que, na condição em que todo o turno de trabalho (8 horas consecutivas) fica com IBUTG acima do limite de tolerância para atividade moderada, a frequência de ocorrência é baixa, ficando entre 0 e 3\% em todo o Estado de São Paulo (Figura 3k).

Entre todas as situações verificadas, o maior risco de sobrecarga térmica, com valores de frequência entre 18 e 21\%, também é atribuído para o norte do estado (Figura 2c). Esse é o caso em que o IBUTG ultrapassa o limite de tolerância para atividade pesada durante 1 hora. Ainda considerando o período de 1 hora de atividade pesada, as demais áreas do estado também mostram frequência alta, em que 9 a $18 \%$ dos dias apresentam valor de IBUTG maior que o limite de tolerância. Computando períodos maiores ( 2 a 8 horas - Figuras 2f, 2i, 2l, 3c, 3f, 3i e 3l) com o IBUTG acima do limite de tolerância para atividade pesada, nota-se que a frequência de ocorrência diminui gradativamente. Porém, é bastante claro que os maiores valores de frequência geralmente ocorrem no centro, norte e oeste do Estado de São Paulo. Para as situações de 8 horas consecutivas com IBUTG acima do limite de tolerância para atividade pesada, a frequência de ocorrência é menor, ficando entre 6 e $9 \%$ no norte de São Paulo.

Referenciando novamente as informações contidas em http://www.dsr.inpe.br/laf/canasat/cultivo.html, que mostram as áreas de safra do cultivo de cana-de-açúcar no Estado de São Paulo, nota-se que as áreas onde predominantemente há cultura de cana-de-açúcar são coincidentes com as áreas de maior risco de estresse térmico (Figuras 2 e 3 ).

Evidentemente que há uma gradativa diminuição da frequência de dias com IBUTG ultrapassando os limites de tolerância, conforme aumenta o número de horas em que o IBUTG é extrapolado (Figura 4). No entanto, considerando as áreas mais críticas de São Paulo em termos de sobrecarga térmica (máxima frequência na Figura 4), percebemos que o trabalhador que desenvolve atividade leve estará exposto a 1 hora com IBUTG acima do limite de tolerância com uma frequência de 7,8\% dos dias de trabalho (Figura 4a). Contudo, ainda considerando atividade leve no local de maior exposição à sobrecarga térmica, o risco do IBUTG ultrapassar o limite de tolerância durante todo o turno de trabalho (8 horas) é nulo (Figura 4a). No caso de atividade moderada, a situação mais provável (frequência de 15,59\%) é a de o trabalhador ficar exposto a 1 hora com IBUTG acima do limite de tolerância (Figura 4b). Já para atividade pesada, que inclui o corte da cana-de-açúcar, o trabalhador estará sujeito a uma frequência de $19,49 \%$ de dias em que estará exposto durante 1 hora a valores de IBUTG acima do limite de tolerância (Figura 4c).

Nas áreas de São Paulo com maior exposição à sobrecarga térmica, a situação em que todo o turno de trabalho (8 horas) registra IBUTG acima do limite de tolerância ocorre com uma frequência de $7,07 \%, 3,29 \%$ e 0,07\% para as atividades pesada, moderada e leve, respectivamente (Figura 4). Essa é uma situação muito crítica em que, durante todo o turno de trabalho (8 horas), os valores de IBUTG ultrapassam os limites de tolerância previstos em lei. É importante ressaltar que, de acordo com os limites apresentados na Tabela 1, nessas situações, o trabalho passa a não ser permitido sem que se adote o regime trabalho descanso e algumas medidas preventivas.

As duas situações marcadas com círculo na Figura 5 ocorreram nas áreas de maior risco de sobrecarga térmica para atividade do tipo pesada, considerando as situações em que o IBUTG ultrapassa o limite de tolerância durante 1 hora (ponto 1) e durante 8 horas (ponto 2). Os gráficos na Figura 5 mostram a evolução do IBUTG no dia em que o seu valor foi o mais alto em toda a série histórica analisada. Nota-se que, nos dois pontos analisados, o valor de IBUTG ultrapassa os limites de tolerância para atividades leve e moderada $\left(25^{\circ} \mathrm{C}\right.$ e $26,7^{\circ} \mathrm{C}$, respectivamente) durante todo o turno de trabalho. Também, nesses dois pontos, o IBUTG se igualou ou ultrapassou o limite de tolerância para atividade pesada $\left(30^{\circ} \mathrm{C}\right)$ em 6 das 8 horas, sendo que, no ponto 1 , o valor máximo do IBUTG chega a $34,2^{\circ} \mathrm{C}$ às 
Figura 2

Frequência de dias com Índice de Bulbo Úmido - Termômetro de Globo (IBUTG) acima do limite de tolerância durante períodos de 1, 2, 3 e 4 horas.

2a)

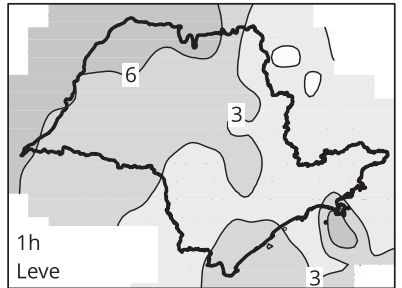

2d)

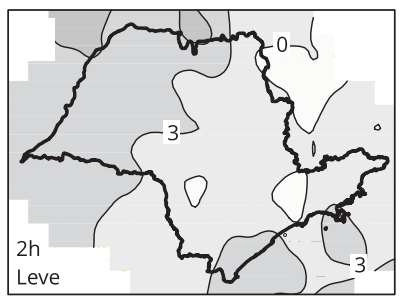

2g)

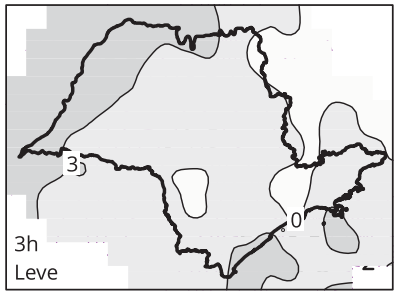

2j)

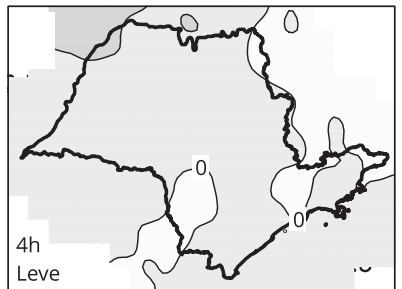

2b)

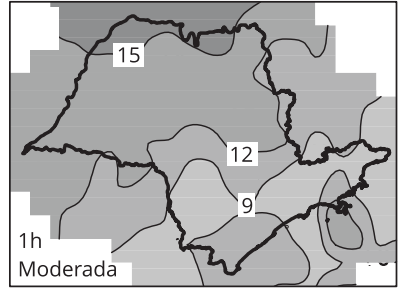

2e)

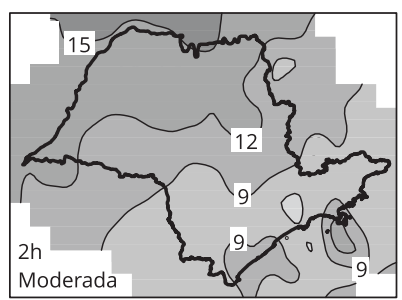

2h)

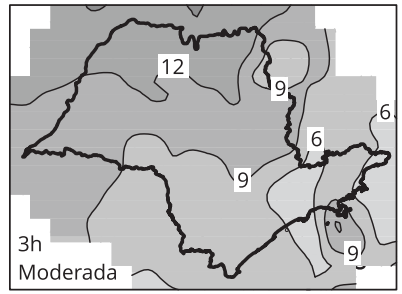

$2 k$ )

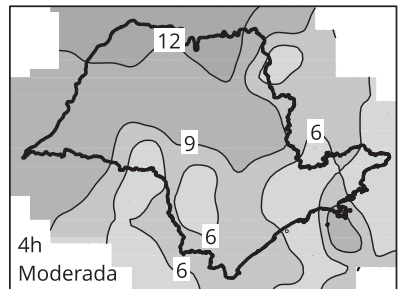

2c)

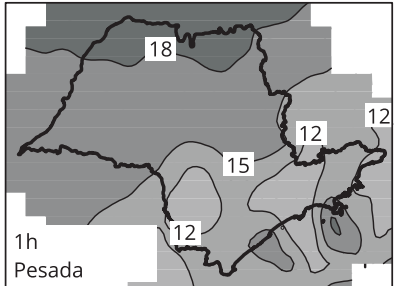

Frequência (\%)

27

24

21

18

2

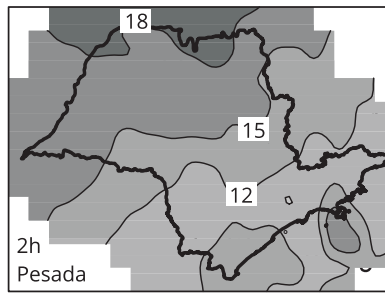

15

12

9

6

3

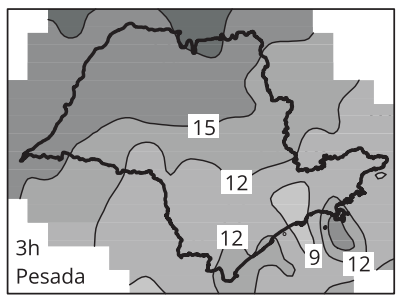

21)

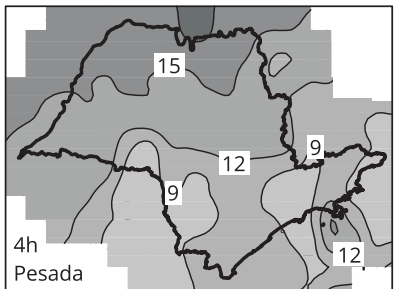

Nota: o período e o tipo de atividade são indicados no canto inferior/esquerdo de cada painel, sendo os respectivos limites de tolerância dados por: $30^{\circ} \mathrm{C}$, para atividade leve, $26,7^{\circ} \mathrm{C}$, para atividade moderada, e $25^{\circ} \mathrm{C}$, para atividade pesada. 


\section{Figura 3}

Frequência de dias com Índice de Bulbo Úmido - Termômetro de Globo (IBUTG) acima do limite de tolerância durante períodos de 5, 6, 7 e 8 horas.

3a)

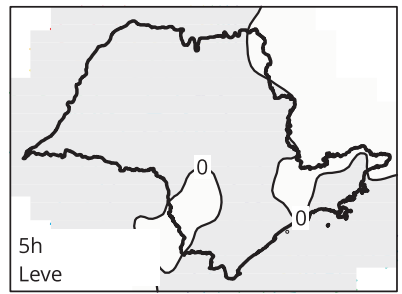

3d)

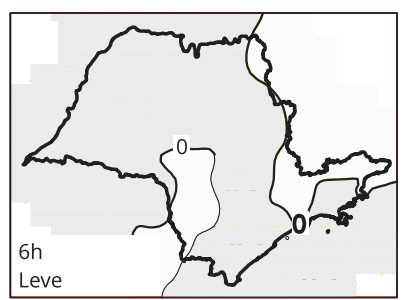

$3 g)$

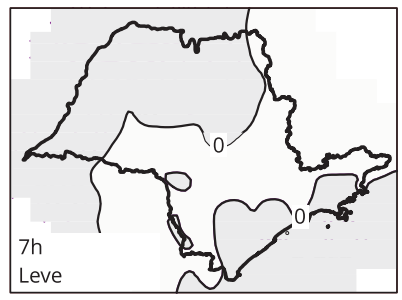

3j)

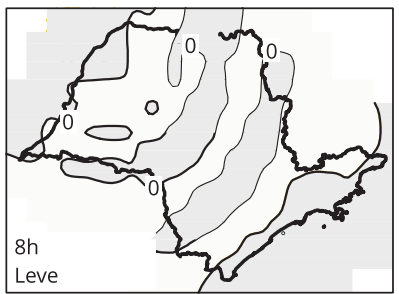

$3 b)$

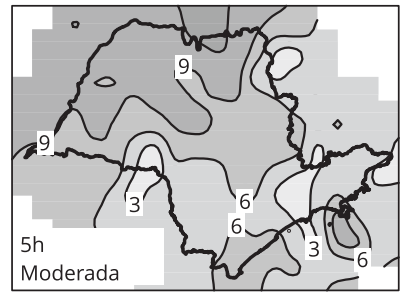

3e)

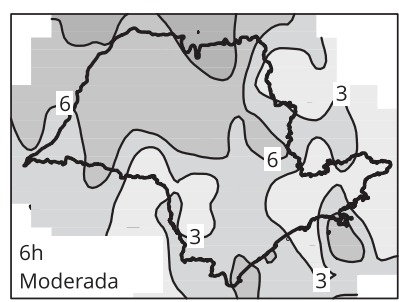

$3 \mathrm{~h})$

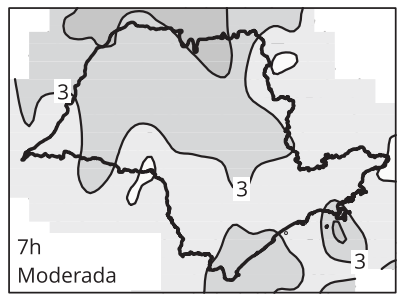

$3 k)$

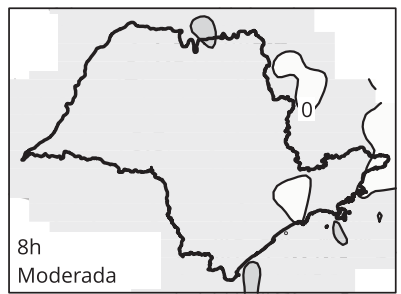

3c)

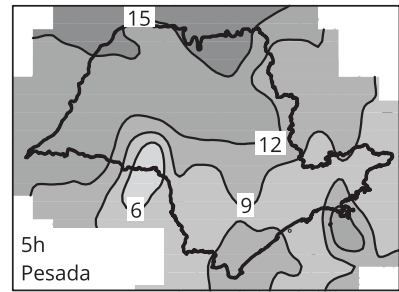

Frequência (\%)

3f)

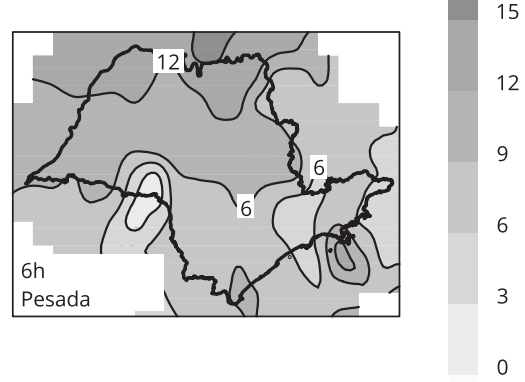

3i)

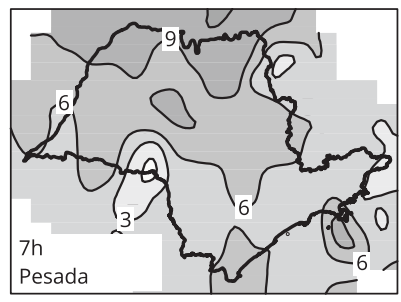

31)

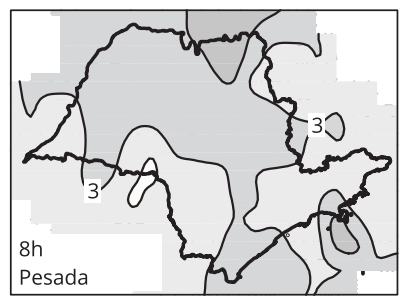

Nota: o período e o tipo de atividade são indicados no canto inferior/esquerdo de cada painel, sendo os respectivos limites de tolerância dados por: $30^{\circ} \mathrm{C}$, para atividade leve, $26,7^{\circ} \mathrm{C}$, para atividade moderada, e $25^{\circ} \mathrm{C}$, para atividade pesada. 
Figura 4

Frequência máxima ( $\mathbf{\Delta}$ ), mínima ( $\mathbf{v}$ ) e média ( $\mathbf{\bullet}$ de dias com Índice de Bulbo Úmido - Termômetro de Globo (IBUTG) acima do limite de tolerância por período de 1 a 8 horas, de acordo com o tipo de atividade.

4a) Leve

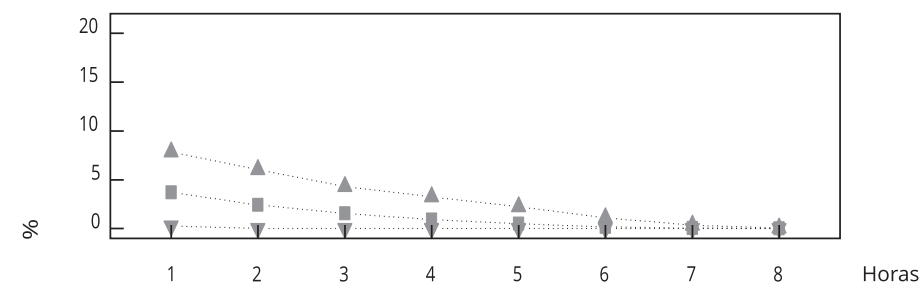

4b) Moderada

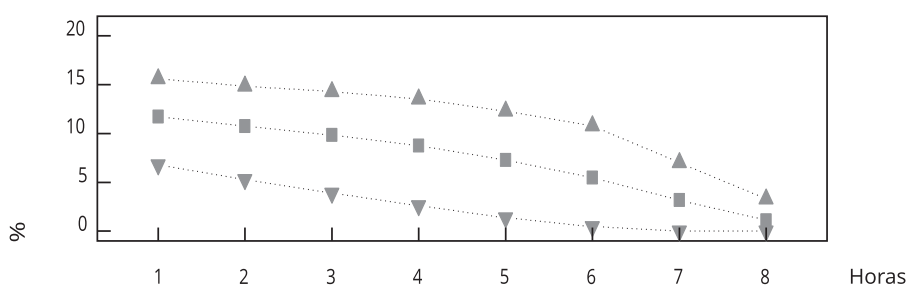

4c) Pesada

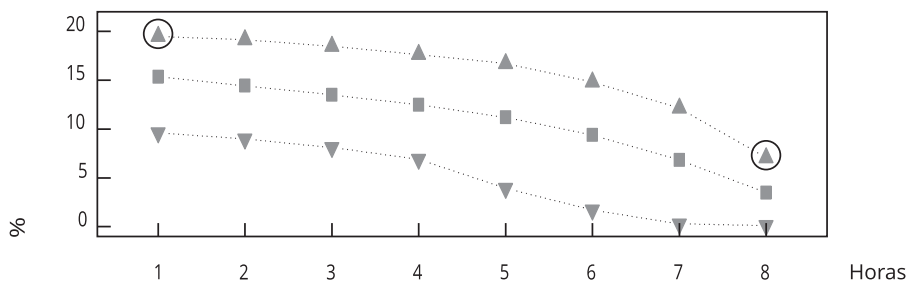

Nota: esses valores de frequência foram obtidos dos 137 pontos para os quais a estimativa do IBUTG foi realizada, e os valores marcados com um círculo no gráfico (4c) ocorreram em localidades do norte do Estado.

14 horas. Nessa situação, sem que medidas especiais sejam adotadas, há exposição a risco grave e eminente. Para tanto, a norma determina a obrigação de adoção de medidas que garantam a integridade da saúde da pessoa exposta. Ressaltamos, ainda, que ambos os pontos, considerados aqui como os que possuem os maiores riscos de exposição à sobrecarga térmica no Estado de São Paulo, localizam-se em uma das áreas de maior atividade da cultura de cana-de-açúcar, conforme informação disponibilizada em http://www.dsr.inpe.br/laf/canasat/cultivo.html. O ponto 2, em especial, faz parte da região mais densamente caracterizada pela cultura. 


\section{Figura 5}

Evolução do Índice de Bulbo Úmido - Termômetro de Globo (IBUTG), entre 10 e 17 horas (horário brasileiro de verão), nos dias 14 de fevereiro de 2013 (ponto 1) e 10 de dezembro de 2010 (ponto 2).
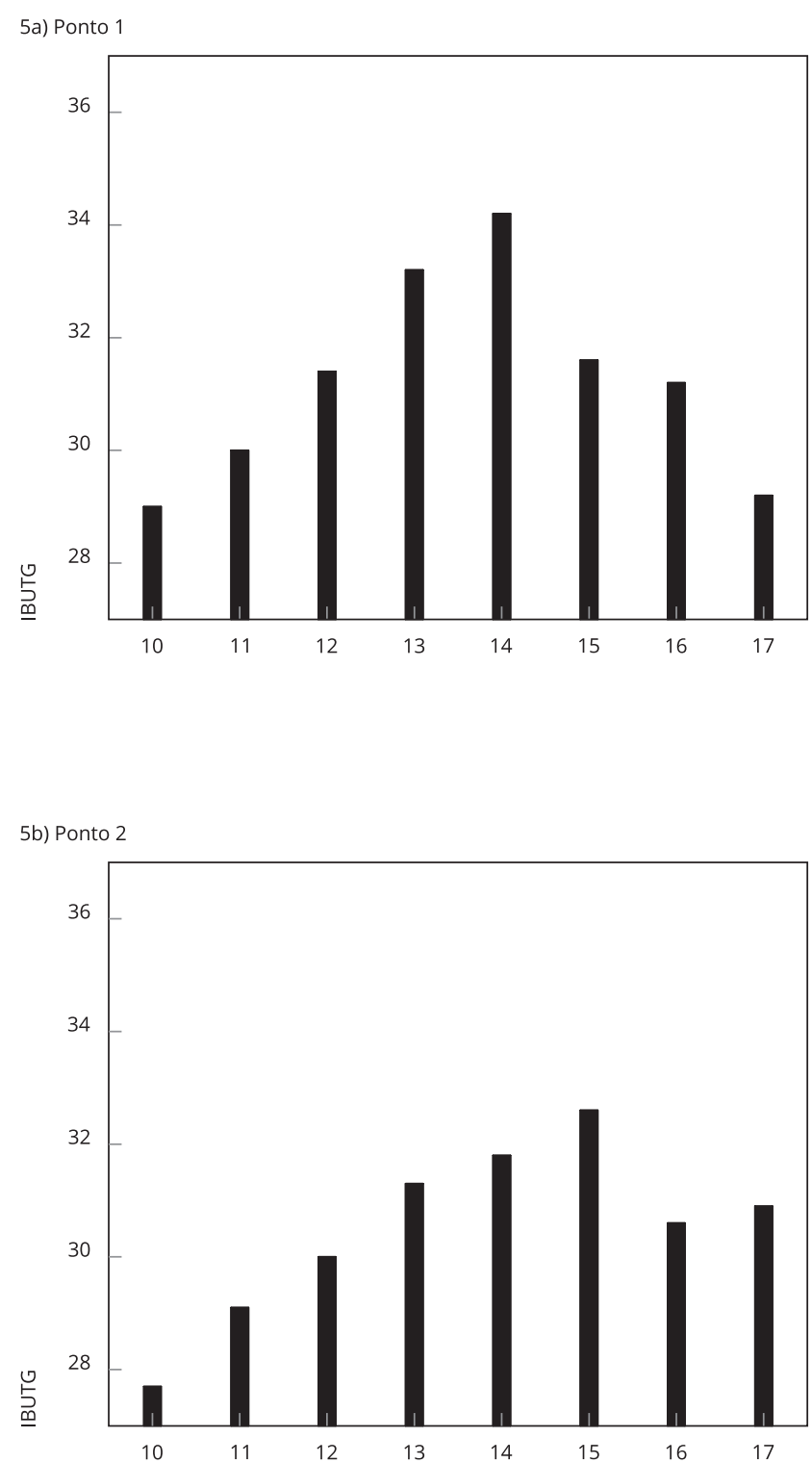

Nota: esses dias são quando ocorreram os maiores valores de IBUTG de toda a série histórica (1ํ de setembro de 2010 a 31 de agosto de 2014).

\section{Considerações finais}

Vários fatores devem ser considerados na avaliação dos efeitos do calor à saúde dos trabalhadores, tais como as vestimentas e a vulnerabilidade socioeconômica do indivíduo. A desconsideração desses fatores é um limite aos resultados de risco provenientes do estresse térmico, tais como os apresentados neste estudo. Contudo, a combinação dos fatores atividade física e calor ambiental colabora de 
forma sinergética e se apresenta como um importante papel para o aumento do risco de agravos à saúde. Neste estudo, analisou-se o risco de sobrecarga térmica por meio do cálculo de frequência de dias com valores de IBUTG acima dos limites previstos em lei, sob a consideração das características de trabalho dos trabalhadores da cultura de cana-de-açúcar no Estado de São Paulo. O IBUTG foi estimado a partir de dados meteorológicos, por meio de metodologia desenvolvida especificamente para São Paulo.

Os resultados deste estudo revelaram considerável frequência de dias com IBUTG elevado, justamente nas áreas de maior produção de cana-de-açúcar. Os valores de IBUTG ultrapassam os limites de tolerância para os três tipos de atividade: leve, moderada e pesada. Nas áreas de São Paulo com maior exposição à sobrecarga térmica, em 1 das 8 horas de trabalho, o IBUTG ultrapassa o limite de tolerância em cerca de $20 \%$ dos dias de trabalho para atividade pesada, cerca de $16 \%$ para atividade moderada e cerca de $8 \%$ para atividade leve. A situação em que o IBUTG ultrapassa os limites de tolerância durante todo o turno de trabalho (8 horas) ocorre em cerca de $7 \%$ dos dias de trabalho, para atividade pesada, e em cerca de $3 \%$, para atividade moderada. O risco de essa situação ocorrer para atividade leve é praticamente nulo. No dia mais crítico de toda a série histórica de um dos pontos de maior exposição à sobrecarga térmica, o valor máximo para o IBUTG alcançou $34,2^{\circ} \mathrm{C}$.

Os resultados apresentados neste estudo são potencialmente importantes de serem considerados no planejamento de políticas públicas e/ou adequações de normas trabalhistas, especialmente aquelas voltadas para trabalhadores que exercem atividades pesadas a céu aberto, como é o caso dos trabalhadores da cana-de-açúcar. Essa temática merece maior atenção ainda ao considerarmos os cenários futuros de mudanças climáticas. Com o advento do aquecimento global, períodos com excessivo calor devem passar a serem mais comuns no Brasil, tendendo a agravar o risco de exposição à sobrecarga térmica.

\section{Colaboradores}

R. C. Roscani contribuiu com a análise e interpretação dos dados, redação do artigo e aprovação final da versão a ser publicada; possui responsabilidade por todos os aspectos do trabalho para a garantia da exatidão e integridade de qualquer parte da obra. D. P. Bitencourt, P. A. Maia e A. C. Ruas contribuíram com a concepção e projeto do estudo, revisão crítica relevante do conteúdo intelectual e aprovação final da versão a ser publicada; possuem responsabilidade por todos os aspectos do trabalho para a garantia da exatidão e integridade de qualquer parte da obra.

\section{Agradecimentos}

Os autores agradecem ao Conselho Nacional de Desenvolvimento Científico e Tecnológico (processos 404201/2013-0 e 449208/2014-1) pelo apoio financeiro, ao programa de pós-graduação da FUNDACENTRO por toda a estrutura e conhecimento transmitido no decorrer da pesquisa, ao Instituto Nacional de Meteorologia (INMET) pelos dados meteorológicos e ao Sr. Norisvaldo Ferraz Júnior pela ajuda nos procedimentos de obtenção das estimativas do IBUTG no software Sobrecarga Térmica. 


\section{Referências}

1. Ministério da Agricultura. Culturas: Cana-deaçúcar. http://www.agricultura.gov.br/vege tal/culturas/cana-de-acucar (acessado em 22/ $\mathrm{Abr} / 2014)$.

2. Instituto Brasileiro de Geografia e Estatística. Levantamento sistemático da produção agrícola. ftp://ftp.ibge.gov.br/Producao_Agricola/ Levantamento_Sistematico_da_Producao_ Agricola_[mensal]/Fasciculo/lspa_201510.pdf (acessado em 22/Abr/2014).

3. Agência Embrapa de Informação e Tecnologia. Árvore do conhecimento cana-de-açúcar.http:// www.agencia.cnptia.embrapa.br/gestor/ca na-de-acucar/arvore/CONTAG01_68_711 200516719.html (acessado em 22/abr/2014).

4. São Paulo. Lei no 11.241 , de 19 de setembro de 2002. Dispõe sobre a eliminação gradativa da queima da palha da cana-de-açúcare dá providências correlatas. Diário Oficial do Estado de São Paulo 2002; 20 set.

5. Vilela RAG, Laat EF, Luz VG, Silva AJN, Takahashi MAC. Pressão por produção e produção de riscos: a "maratona" perigosa do corte manual da cana-de-açúcar. Rev Bras Saúde Ocup 2015; 40:30-48.

6. Gomes JR, Fischer FM, Bedricow B. Trabalho da criança e do adolescente no corte da cana de açúcar. Rev Bras Saúde Ocup 2001; 26:9-27.

7. Dutra FNX. Exploração do trabalho infantojuvenil nas usinas de açúcar e álcool do Estado de Alagoas. Revista Jus Navigandi 2010; ano 15. https://jus.com.br/artigos/17713.

8. Alves F. Por que morrem os cortadores de cana? Saúde Soc 2006; 15:90-8.

9. Tatsch JD. Uma análise dos fluxos de superfície e do microclima sobre cerrado, cana-de-açúcar e eucalipto, com implicações para mudanças climáticas regionais [Dissertação de Mestrado]. São Paulo: Universidade de São Paulo; 2006.

10. Ribeiro H. Queimadas de cana-de-açúcar no Brasil: efeitos à saúde respiratória. Rev Saúde Pública 2008; 42:370-6.

11. Cançado JEDA. Poluição atmosférica e sua relação com a saúde humana na região canavieira de Piracicaba-SP [Tese de Doutorado]. São Paulo: Universidade de São Paulo; 2003.

12. Bitencourt DP, Ruas AC, Maia PA. Análise da contribuição das variáveis meteorológicas no estresse térmico associada à morte de cortadores de cana-de-açúcar. Cad Saúde Pública 2012; 28:65-74.

13. Juttel LP. Esforço físico excessivo busca aumento de renda. Ciênc Cult 2008; 60:6-7.

14. Alessi NP, Navarro VL. Saúde e trabalho rural: o caso dos trabalhadores da cultura canavieira na região de Ribeirão Preto, São Paulo, Brasil. Cad Saúde Pública 1997; 13 Suppl 2:111-21.

15. Ferreira LL, Gonzaga MC, Donatelli S, Bussacos MA. Análise coletiva do trabalho dos cortadores de cana da região de Araraquara, São Paulo. São Paulo: Fundacentro; 2008.
16. Rumin CR, Navarro VL, Perioto NW. Trabalho e saúde no agrobusiness paulista: estudo com colhedores manuais de cana-de-açúcar da região oeste do Estado de São Paulo. Cad Psicol Soc Trab 2008; 11:193-207.

17. Barbosa CMG. Avaliação cardiovascular e respiratória em um grupo de trabalhadores cortadores de cana-de-açúcar queimada no Estado de São Paulo SP [Tese de Doutorado]. São Paulo: Universidade de São Paulo; 2011.

18. Xiang J, Bi P, Pisaniello D, Hansen A. Health impacts of workplace heat exposure: an epidemiological review. Ind Health 2014; 52:91-101.

19. Jay O, Kenny GP. Heat exposure in the Canadian workplace. Am J Ind Med 2010; 53:842-53.

20. Laat EF. Trabalho e risco no corte manual de cana-de-açúcar: a maratona perigosa nos canaviais [Tese de Doutorado]. Piracicaba: Universidade Metodista de Piracicaba; 2010.

21. Ruas AC. Conforto térmico nos ambientes de trabalho. São Paulo: Fundacentro; 1999.

22. Lamberts R, Xavier AA, Goulart S, Vecchi R. Conforto térmico e stress térmico. Florianópolis: Laboratório de Eficiência Energética em Edificações; 2011.

23. Tawatsupa B, Lim LL, Kjellstrom T, Seubsman SP, Sleigh A. Association between occupational heat stress and kidney disease among 37,816 workers in the Thai Cohort Study (TCS). J Epidemiol 2012; 22:251-60.

24. Barbosa CMG, Terra-Filho M, Albuquerque ALP, Di Giorgi D, Grupi C, Negrão CE, et al. Burnt sugarcane harvesting - cardiovascular effects on a group of healthy workers, Brazil. PLoS One 2012; 7:e46142.

25. Sett M, Sahu S. Effects of occupational heat exposure on female brick workers in West Bengal, India. Glob Health Action 2014; 7:1-11.

26. Bethel JW, Harger R. Heat-related Illness among Oregon farm workers. Int J Environ Res Public Health 2014; 11:9273-85.

27. Mirabelli MC, Richardson DB. Heat-related fatalities in North Carolina. Am J Public Health 2005; 95:635-7.

28. Basu R, Samet JM. Relation between elevated ambient temperature and mortality: a review of then epidemiologic evidence. Epidemiol Rev 2002; 24:190-202.

29. Todd N, Valleron AJ. Space-time covariation of mortality with temperature: a systematic study of deaths in France, 1968-2009. Environ Health Perspect 2015; 123:659-64.

30. Hyatt OM, Lemke B, Kjellstrom T. Regional maps of occupational heat exposure: past, present, and potential future. Glob Health Action 2010; 3:1-10.

31. Paixão EDJ, Nogueira PJ. Efeitos de uma onda de calor na mortalidade. Rev Port Saúde Pública $2003 ; 21: 41-54$. 
32. Crowe J, Moya-Bonilla JM, Román-Solano B, Robles-Ramírez A. Heat exposure in sugarcane workers in Costa Rica during the non-harvest season. Glob Health Action 2010; 3:1-9.

33. Yaglou CP, Minard D. Control of heat casualties at military training centers. AMA Arch Ind Health 1957; 16:302-16.

34. Ministério do Trabalho e Emprego. NR-15 atividades e operações insalubres. http://por tal.mte.gov.br/images/Documentos/SST/NR/ NR15/NR15-ANEXO3.pdf (acessado em 18/ Fev/2013).

35. International Organization Standardization. ISO 7243: hot environments: estimation of the heat stress on working man. Geneva: International Organization Standardization; 1989.

36. Kjellstrom T, Lemke B, Otto M. Mapping occupational heat exposure and effects in SouthEast Asia: ongoing time trends 1980-2011 and future estimates to 2050. Ind Health 2013 ; 51:56-67.

37. Ministério do Trabalho e Emprego. NR-09 Programa de Prevenção de Riscos Ambientais. http://portal.mte.gov.br/images/Documentos/ SST/NR/NR9.pdf (acessado em 18/Fev/2013).

38. Dash SK, Kjellstrom T. Workplace heat stress in the context of rising temperature in India. Curr Sci 2011; 101:496-503.

39. Dernedde TE, Gilbert D. Prediction of wetbulb globe temperatures in aluminum smelters. Am Ind Hyg Assoc J 1991; 52:120-6.

40. Bernard TE, Pourmoghani M. Prediction of workplace wet bulb global temperature. Appl Occup Environ Hyg 1999; 2:126-34.

41. Hunter $\mathrm{CH}$, Minyard CO. Estimating wet bulb globe temperature using standard meteorological measurements. In: 2nd Symposium on Environmental Applications. Boston: American Meteorological Society; 2010. p. 9-14.
42. Matthew WT, Santee WR, Berglund LG. Solar load inputs for thermal strain models and the solar radiation sensitive components of the WBGT Index. http://www.dtic.mil/dtic/ tr/fulltext/u2/a392480.pdf (acessado em 20/ $\mathrm{Mai} / 2014)$

43. Tonouchi M, Murayama K, Ono M. WBGT forecast for prevention of heat stroke in Japan. In: Sixth Symposium on the Urban Environment. AMS Forum: Managing our Physical and Natural Resources: Successes and Challenges; 2006. https://ams.confex.com/ams/An nual2006/techprogram/paper_101722.htm.

44. Liljegren JC, Carhart RA, Lawday P, Tschopp S, Sharp R. Modeling wet bulb globe temperature using standard meteorological measurements. J Occup Environ Hyg 2008; 10:645-55.

45. Gaspar AR, Quintela DA. Physical modelling of globe and natural wet bulb temperatures to predict WBGT heat stress index in outdoor environments. Int J Biometeorol 2009; 53:221-30.

46. Lemke B, Kjellstrom T. Calculating workplace WBGT from meteorological data: a tool for climate change assessment. Ind Health 2012; 50:267-78.

47. Patel T, Mullen SP, Santee WR. Comparison of methods for estimating wet-bulb globe temperature index from standard meteorological measurements. Mil Med 2013; 178:926-33.

48. Maia PA, Ruas AC, Bitencourt DP. Wet-bulb globe temperature index estimation using meteorological data from São Paulo State, Brazil. Int J Biometeorol 2015; 59:1395-403.

49. International Organization Standardization. ISO 7243: ergonomics of the thermal environmental - determination of metabolic. Geneva: International Organization Standardization; 2004. 


\section{Abstract}

Environmental heat is an aggravating factor for sugarcane workers, and manual cane cutting is one of the heavy work activities that favor the generation of metabolic heat. This can combine with other precarious working conditions to produce heatstroke, potentially leading to serious complications and even death. The study aimed to quantify the risk of overheating in sugarcane cutters in São Paulo, Brazil. Drawing on a specific methodology, the study used four years of data to estimate the Globe Thermometer Wet Bulb Index based on light, moderate, and heavy activities. The results showed that the legal limit was exceeded for continuous activity in periods from 1 to 8 hours, which requires control measures in order to continue working. The principal observation was the risk of heatstroke in the three types of activity: light, moderate, and heavy. The limits were exceeded throughout the work shift on $7 \%$ of the days for heavy activity and on 3\% of the days for moderate activity. In addition, the areas with the highest risk of heatstroke coincide with predominant cane-growing.

Heat Stress Disorders; Rural Workers; Saccharum

\section{Resumen}

El calor ambiental es un agravante para las actividades de los trabajadores de plantaciones de caña de azúcar, siendo el corte manual una de las actividades pesadas que favorece la elevada generación de calor metabólico. Esto, asociado con otras condiciones precarias de trabajo, favorece el estrés térmico, pudiendo provocar graves enfermedades o incluso llevar a la muerte. El objetivo de este estudio es cuantificar el riesgo de sobrecarga térmica a la que los cortadores de caña de azúcar de São Paulo, Brasil, están expuestos. Mediante una metodología específica, se utilizaron cuatro años de datos para estimar el Índice de Bulbo Húmedo-Termómetro de Globo, considerando a las actividades como: leves, moderadas y pesadas. Los resultados muestran la extrapolación del limite legal de exposición para la actividad continua en períodos de 1 a 8 horas, lo que requiere medidas de control para la continuidad del trabajo. La principal constatación es la observancia del riesgo de sobrecarga térmica para los tres tipos de actividad: leve, moderada y pesada. Los limites de tolerancia se sobrepasan durante todo el turno de trabajo en cerca de un 7\% dos dias, en el caso de la actividad pesada, $y$ en cerca de un 3\% en la actividad moderada. Además, se percibió que las áreas con mayor riesgo de sobrecarga térmica son las mismas donde hay predominancia de plantaciones de caña de azúcar.

Trastornos de Estrés por Calor; Trabajadores Rurales; Saccharum
Recebido em 23/Dez/2015

Versão final reapresentada em 10/Mai/2016

Aprovado em 13/Jun/2016 